Chirurg 2015 $\cdot 86: 707$

DOI 10.1007/s00104-015-0036-6

Online publiziert: 20. Juni 2015

(c) Springer-Verlag Berlin Heidelberg 2015

CrossMark

H. Dralle $\cdot$ I. Satiroglu

Universitätsklinik für Allgemein-, Viszeral- und Gefäßchirurgie, Medizinische Fakultät,

Universitätsklinikum Halle (Saale), Halle (Saale), Deutschland

\title{
Inzidentelles Appendixkarzinoid im Kindesalter
}

\section{Ergebnisse}

Insgesamt 13 der 28 Kinder (46\%) hatten eine histologisch verifizierte Appendizitis (10 unkompliziert, 3 perforiert). Die mediane Tumorgröße betrug $0,7 \mathrm{~cm}$ (Range $0,2-1,6 \mathrm{~cm}$ ), bei nur 4 Kindern lag sie $>1 \mathrm{~cm} .22$ Appendixkarzinoide hatten eine Ausdehnung in die Submukosa (3), Muscularis propria (1), Subserosa (5), Serosa (6) oder Mesoappendix (7); in allen Fällen handelte es sich um eine R0-Resektion. Bei 2 von 5 nachoperierten Kindern (1 Ileozökalresektion, 4 Hemikolektomien) wurde im Resektat Resttumor (1 Zökalmesenterium, 1 Lymphknotenmetastase [1/43]) nachgewiesen. Die Nachoperationen verliefen komplikationslos. Bei keinem der nachkontrollierten Kinder erfolgte eine weitere Operation, keines der Kinder hatte ein Rezidiv.

\section{Diskussion und Fazit}

Ob die allgemeinen Behandlungsempfehlungen für Erwachsene mit inzidentellem Appendixkarzinoid ([1, 2]; Nachoperation bei Primärtumoren $>2 \mathrm{~cm}$, Mesoappendixinfiltration, Goblet-Zelloder Adenokarzinoid, R1-Appendektomie; keine Nachkontrolle bei Low-gradeAppendixkarzinoiden ohne Metastasen und $<1 \mathrm{~cm}$ ) auch für Kinder gelten, ist unklar. Die vorliegende Studie stellt dieses Vorgehen unter Einbezug eines aktuellen Literaturreviews infrage und begründet dies mit der geringen Ausdehnung und Aggressivität der Tumoren im Kindesalter.

Die Studie hat eine Reihe von Limitationen (z. B. keine Angabe zur Korrelation von Tumorgröße und Invasionstiefe; keine Angabe der Indikationen zur Nachoperation; kurze Nachbeobachtungs- zeit bei nur zwei Drittel der Kinder). Allerdings betonen die Autoren zu recht, dass nicht nur bez. der Indikation zur Nachoperation, sondern auch bez. der Art und Methoden zur bildgebenden und laborchemischen Nachkontrolle dringender Bedarf an systematischen Untersuchungen besteht, um daraus Behandlungsempfehlungen zum Appendixkarzinoid im Kindesalter ableiten zu können.

\section{Korrespondenzadresse}

Prof. Dr. med. Dr. h. c H. Dralle FRCS, FACS, FEBS

Universitätsklinik für Allgemein-, Viszeral- und Gefäßchirurgie, Medizinische Fakultät Universitätsklinikum Halle (Saale) Ernst-Grube-Straße 40, 06097 Halle (Saale) henning.dralle@uk-halle.de

Einhaltung ethischer Richtlinien

Interessenkonflikt. H. Dralle und I. Satiroglu geben an, dass kein Interessenkonflikt besteht.

\section{Literatur}

1. Pape UF, Perren A, Niederle B, Gross D, Gress T, Costa F, Arnold R, Denecke T, Plockinger U, Salazar R, Grossman A, Barcelona Consensus Conference participants (2012) ENETS Consensus Guidelines for the management of patients with neuroendocrine neoplasms from the jejuno-ileum and the appendix including goblet cell carcinomas. Neuroendocrinology 95:135-156

2. Dralle H (2011) Chirurgische Strategien beim Zufallsbefund eines Appendixkarzinoids. Chirurg 82:598-606 